\title{
Hérnia umbilical primária: melhor manejo operatório no adulto
}

Cirênio de Almeida Barbosa ${ }^{1}$ (D), Deborah Campos Oliveira² (D), Marcela de Matos Assunção ${ }^{3}$ (D), Mariana Fonseca Guimarães ${ }^{3} \mathbb{1}$

\section{RESUMO}

Modelo do estudo: Revisão sistemática. Objetivo: Avaliar se é facultativo ou imprescindível o uso de malha cirúrgica no reparo das pequenas hérnias umbilicais primárias, com orifício menor que $2 \mathrm{~cm}$, a fim de oferecer melhores evidências aos cirurgiões e, assim, aprimorar o método cirúrgico e o seu desfecho. Métodos: Trata-se de uma revisão da literatura, cuja busca foi direcionada aos artigos que abordassem o manejo operatório das hérnias abdominais, sobretudo das hérnias umbilicais de pequeno tamanho. A pesquisa foi realizada nas bases de dados primárias PubMed, LILACS, Cochrane Library e Periódicos CAPES. Resultados: No total, foram incluídos quatro estudos. Foram avaliadas as taxas de recorrência, bem como as de complicações pós-operatórias após a correção da hérnia umbilical com e sem o uso de tela, observando-se o tamanho do defeito abdominal. Foi observada diminuição da recorrência das hérnias após o reparo com tela. No entanto, complicações, como infecção da ferida operatória, foram mais comumente observadas com o uso da prótese. Não houve consenso quanto ao uso da tela em hérnias menores que $1 \mathrm{~cm}$. Conclusão: O uso de próteses pode vir a ser o tratamento de escolha no reparo das hérnias umbilicais primárias. Contudo, mais estudos são necessários para avaliar o papel dessa estratégia no manejo das hérnias menores que $1 \mathrm{~cm}$.

Palavras-chave: Hérnia Umbilical; Parede Abdominal; Telas Cirúrgicas.

1 Universidade Federal de Ouro Preto, Escola de Medicina, Departamento de Cirurgia, Ginecologia e Obstetrícia e Propedêutica, Ouro Preto, (MG), Brasil.

2 Universidade Federal de Ouro Preto, Ouro Preto (MG), Brasil.

3 Universidade Federal de Ouro Preto, Escola de Medicina, Ouro Preto, (MG), Brasil. 


\section{INTRODUÇÃO}

A hérnia umbilical é uma condição cirúrgica de alta prevalência na população e, diante da sua identificação, a correção eletiva é recomendada. Sabe-se que o seu reparo por sutura simples tem taxas de recorrência inaceitavelmente altas, sendo relatadas, em algumas séries de estudos, taxas de até $54 \%$, sendo que estudos mais recentes encontraram taxas de até $14 \%{ }^{1}$, o que torna esse tipo de reparo pouco resolutivo. Diante disso, tem sido cada vez mais frequente na literatura médica a discussão acerca dos benefícios da aplicação de telas no reparo das hérnias umbilicais, assim como é realizado para as demais hérnias de parede abdominal, e as evidências sugerem que o reparo com malhas é recomendado ${ }^{2,3}$. A tela cirúrgica tem sua principal indicação fundamentada no fato de diminuir a possibilidade ou evitar recidivas, dor e tensão excessiva no local do defeito da parede abdominal.

As malhas podem ser aplicadas por abordagens cirúrgicas abertas ou laparoscópicas, sendo que ambas as técnicas apresentam bons resultados clínicos ${ }^{4-6}$. Entretanto, a colocação por laparoscopia, ao promover descontinuidades nas fáscias dos músculos abdominais e, com isso, gerar vários novos pontos de fraqueza na parede do abdome, apresenta certa desvantagem. Essa dificuldade, contudo, funcionou como acelerador para o desenvolvimento dos "patches", pequenos dispositivos que podem ser inseridos e fixados abaixo do peritônio e que não produzem novos defeitos nas fáscias abdominais ${ }^{7-9}$. Os "pedaços de tela" parecem ser especialmente adequados para o reparo das hérnias de pequeno tamanho, já que requerem menor dissecção de tecido para sua colocação, além de estimularem de forma significativa a multiplicação e migração de fibroblastos localmente.

A utilização de telas para reforço do reparo cirúrgico das grandes hérnias da parede abdominal reduz o risco de recorrência de $15-40 \%$ para $1-10 \%$, aproximadamente ${ }^{2,10-15}$. Até o momento, grande parte das hérnias de pequenos orifícios são tratadas por reparo por sutura simples, uma vez que o plano pré-peritoneal, ao qual a tela, em geral, é fixada, é um plano de difícil manuseio nesse cenário. Isso justifica, em parte, o pequeno número de estudos acerca do uso das malhas para o reparo de defeitos da parede com pequenos orifícios herniários. O uso das telas, além de alterar os resultados terapêuticos das hérnias umbilicais e epigástricas cujos orifícios são pequenos, pode ter implicações positivas também nas hérnias incisionais de tamanho inferior a $2 \mathrm{~cm}$, como é o caso das hérnias que se originam no local de inserção dos trocartes nas cirurgias laparoscópicas.

O grau de dificuldade de execução de um procedimento não é um parâmetro de grande relevância clínica, todavia esse fator pode ser determinante na decisão terapêutica do cirurgião: as dificuldades relacionadas à colocação da tela e as possíveis complicações resultantes do seu uso podem ser a justificativa pela qual grande parte das pequenas hérnias abdominais, de qualquer tipo, ainda são reparadas com rafia simples, sem o reforço com tela.

Considerando-se a alta prevalência das hérnias da parede abdominal, em especial as "corriqueiras" hérnias umbilicais pequenas e primárias; o avanço tecnológico na especialidade cirúrgica; a evolução das telas disponíveis para uso; e as altas taxas de recidiva após correção com rafia simples, essa revisão sistemática tem como objetivo avaliar se é facultativo ou necessário o uso da tela no reparo das pequenas hérnias umbilicais em adultos, visando, portanto, proporcionar ao cirurgião decisões baseadas nas melhores evidências disponíveis e, assim, aprimorar o método cirúrgico e o seu desfecho.

\section{METODOLOGIA}

Trata-se de uma revisão sistemática da literatura, conduzida nas bases de dados primárias PubMed, LILACS, Periódicos CAPES e Cochrane Library. Foram utilizadas as palavras chaves "umbilical hernia", "mesh repair" e "suture repair" e a estratégia de busca formulada, "((umbilical hernia) AND (mesh repair)) AND (suture repair)", foi empregada em todas as bases de dados para a recuperação de estudos.

Realizada a pesquisa, os estudos foram cruzados para a identificação e exclusão de duplicatas, para que, então, fosse feita a seleção dos artigos. Esta foi feita a partir do rastreamento através dos títulos e resumos, seguido da leitura crítica do texto na íntegra daqueles estudos considerados possivelmente elegíveis. Além disso, foi feita verificação 
das referências bibliográficas dos artigos relevantes para complementação da pesquisa.

Foram incluídos os estudos de intervenção, do tipo Ensaio Clínico Randomizado (ECR), publicados entre os anos de 2000 e 2020, em língua inglesa e que tinham como objetivo comparar os resultados do reparo das hérnias umbilicais com e sem o uso de telas. Considerou-se como critérios de inclusão estudos realizados com adultos de ambos os sexos, diagnosticados com hérnias umbilicais primárias, submetidos a correções eletivas. Os artigos que incluíssem apenas pacientes com hérnias umbilicais maiores que $3 \mathrm{~cm}$, referentes a hérnias umbilicais encarceradas/estranguladas, recorrentes ou secundárias ou referentes a outros tipos de hérnias foram excluídos, assim como os estudos de outros tipos, capítulos de livros, anais de congresso/outros eventos científicos e artigos não disponíveis na íntegra.

Os seguintes dados foram extraídos de cada estudo: primeiro autor e ano de publicação, tamanho amostral, faixa etária, critérios de inclusão e exclusão, duração do acompanhamento, tamanho do orifício herniário, técnicas de reparo utilizadas e taxas de recorrência e complicações pós-operatórias, como seroma, hematoma e infecção da ferida operatória (IFO).

A pesquisa foi realizada por dois pesquisadores (M.M.A e M.F.G), de forma independente, sendo as discordâncias solucionadas por discussão entre ambos. Ademais, para a análise da qualidade metodológica dos estudos, foi utilizada a Cochrane Risk of Bias Tool. Essa ferramenta permite a avaliação do risco de viés em baixo (low), alto (high) ou incerto (unclear) por meio da caracterização dos seguintes domínios da pesquisa: selection, perfomance, detection, attrition, reporting e other bias ${ }^{16}$ (Tabela 1 ).

A revisão sistemática foi executada de acordo com as recomendações da Preferred Reporting Items for Systematic Review and Meta-Analyses (PRISMA).

Tabela 1

Avaliação da qualidade metodológica dos estudos incluídos. Adaptado de Cochrane Risk of Bias Tool.

\begin{tabular}{lcccc}
\hline & $\begin{array}{c}\text { Arroyo et al. } \\
\mathbf{2 0 0 1}\end{array}$ & $\begin{array}{c}\text { Kaufmann et } \\
\text { al. } \mathbf{2 0 1 8}\end{array}$ & $\begin{array}{c}\text { Khattab et al. } \\
\mathbf{2 0 2 0}\end{array}$ & $\begin{array}{c}\text { Polat et al. } \\
\mathbf{2 0 0 5}\end{array}$ \\
\hline Geração de sequência aleatória & Baixo & Baixo & Incerto & Baixo \\
Ocultação de alocação & Baixo & Baixo & Baixo & Incerto \\
Relato de desfecho seletivo & Baixo & Baixo & Baixo & Baixo \\
Cegamento dos participantes e da equipe & Incerto & Baixo & Incerto & Incerto \\
Cegamento de avaliação do desfecho & Incerto & Baixo & Incerto & Incerto \\
Desfechos incompletos & Baixo & Baixo & Baixo & Baixo \\
Outras fontes de viés & Baixo & Baixo & Baixo & Incerto \\
\hline
\end{tabular}

\section{RESULTADOS}

De um total de 1118 estudos localizados, apenas quatro foram incluídos nessa revisão. Foram excluídos os artigos que se limitavam a analisar apenas o manejo de hérnias umbilicais primárias maiores que $3 \mathrm{~cm}$, complicadas, recorrentes ou secundárias, bem como os que se referiam a outros tipos de hérnias. Estudos de outros tipos, capítulos de livros e anais de congresso/outros eventos científicos também não foram incluídos na análise (Figura 1).
O tamanho da amostra dos estudos selecionados variou entre 50 e 300 pacientes, obtendo-se um total de 650 indivíduos, com idade entre 14 e 82 anos. Foram consideradas, na maioria dos estudos, hérnias umbilicais primárias menores ou iguais a $4 \mathrm{~cm}$, com exceção de um estudo, que não fez restrições quanto ao tamanho do defeito da parede abdominal. Os pacientes foram acompanhados por, no mínimo, 6 meses (Tabela 2).

De acordo com Arroyo et al. (2001), a taxa de recorrência após uso de tela na correção cirúrgica da hérnia umbilical é significativamente menor 
(1\%), quando comparada com a correção por sutura simples $(11 \%)$, independentemente do tamanho do seu orifício. Além disso, nesse estudo, complicações como seroma, hematoma e IFO tiveram incidências semelhantes em ambas as abordagens ${ }^{17}$.

Kaufmann et al. (2018) encontraram uma taxa de recorrência de $4 \%$ após uso de telas na correção cirúrgica dessas hérnias e de $12 \%$ após utilização da estratégia clássica, após um período de 24-30 meses de acompanhamento. Ainda, foi observado que as recorrências se apresentaram mais rapidamente entre os indivíduos submetidos à correção sem tela: as recorrências surgiram após cerca de 3,6 meses após a estratégia clássica, ao passo que, após o uso de telas, foram verificadas após 12,6 meses. No entanto, as hérnias menores que $1 \mathrm{~cm}$ foram excluídas do estudo, devido à dificuldade de inserção da tela, dado o pequeno tamanho do orifício, o que poderia requerer aumento do defeito. Além disso, observou-se uma pequena maior taxa de incidência de IFO no pós-operatório no grupo de pacientes tratados com tela, diferença essa, no entanto, não significativa. Quanto às demais complicações avaliadas, hematoma e seroma, também não foram constatadas diferenças significativas entre as abordagens ${ }^{18}$.

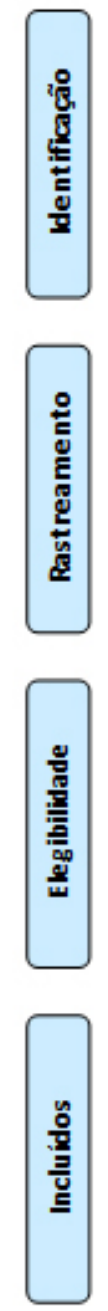

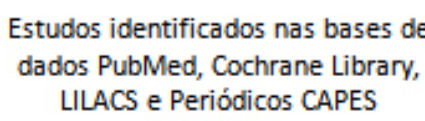

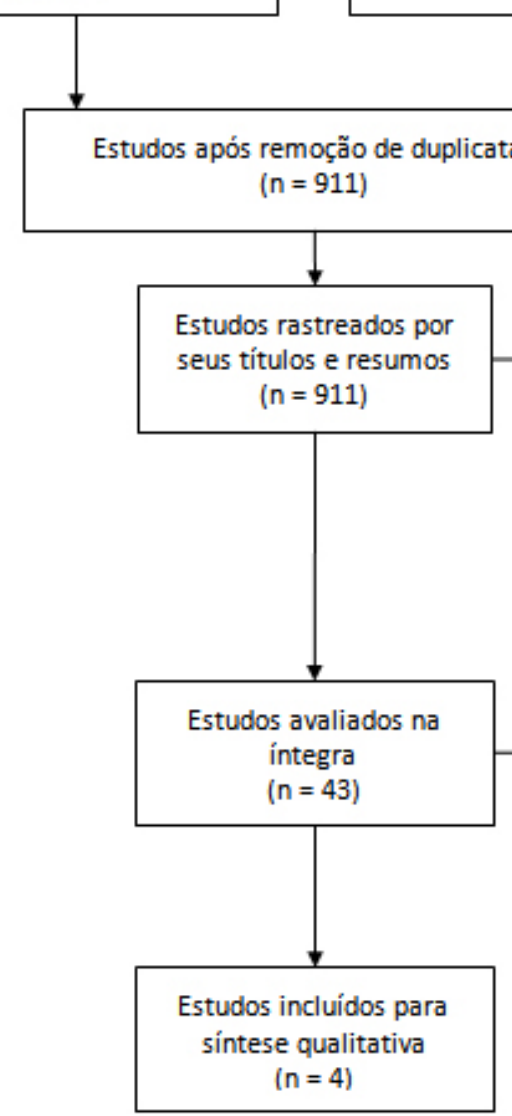

Estudos adicionais identificados por outros meios $(n=5)$

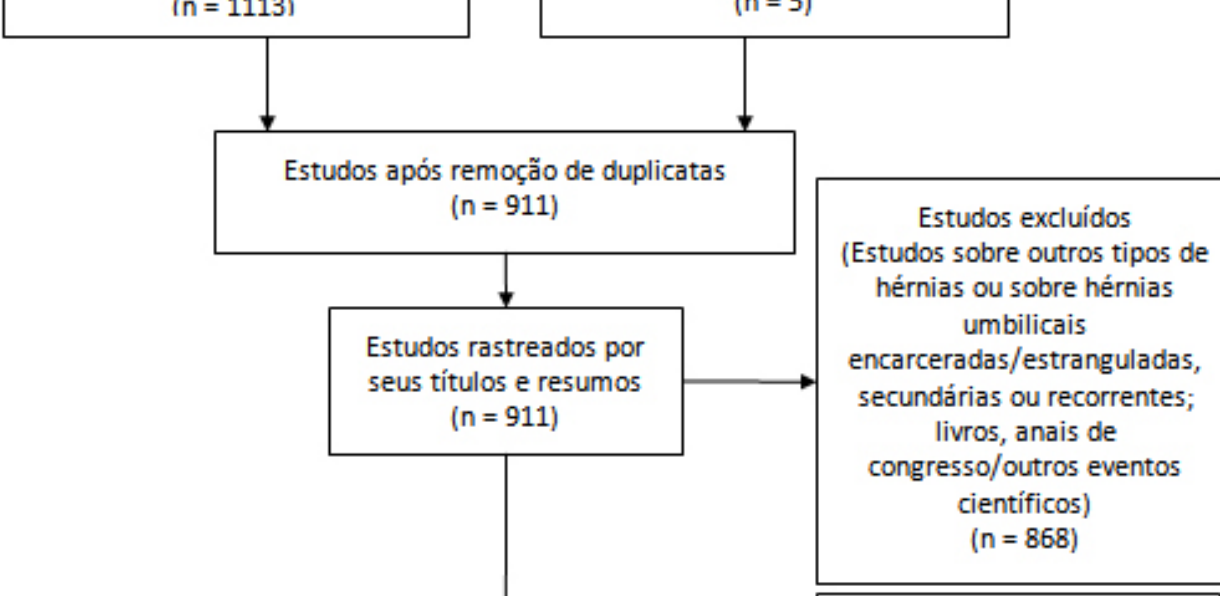

Estudos excluídos (Estudos não disponiveis na íntegra; publicados em outra língua; estudos retrospectivos ou prospectivos observacionais; que incluíam apenas hérnias umbilicais $>3$ $\mathrm{cm}$ ) $(\mathrm{n}=39)$

Figura 1: Fluxograma de sistematização dos estudos incluídos nessa revisão. 
Tabela 2

Características dos estudos incluídos.

\begin{tabular}{|c|c|c|c|c|}
\hline Autor \& ano & Amostra & $\begin{array}{l}\text { Faixa etária } \\
\text { (anos) }\end{array}$ & $\begin{array}{c}\text { Duração do acompanhamento } \\
\text { (meses) }\end{array}$ & $\begin{array}{l}\text { Tamanho do } \\
\text { orifício herniário }\end{array}$ \\
\hline Arroyo et al. 2001 & 200 & 14 a 79 & 64 (21 a 80; média) & $>0 \mathrm{~cm}$ \\
\hline Kaufmann et al. 2018 & 300 & 20 a 77 & 25,1 ( 0 a 87,$8 ;$ mediana) & $1-4 \mathrm{~cm}$ \\
\hline Khattab et al. 2020 & 100 & 27 a 55 & 6 & $<3 \mathrm{~cm}$ \\
\hline Polat et al. 2005 & 50 & 27 a 82 & 22 (6 a 44; média) & $<4 \mathrm{~cm}$ \\
\hline
\end{tabular}

Ao contrário do observado nos estudos supracitados, Polat et al. (2005) constataram que a ocorrência de complicações e de recorrência foi similar entre os indivíduos submetidos à correção com tela e os submetidos à correção sem tela. Vale ressaltar que duas recorrências foram detectadas após a utilização da sutura simples, ao passo que nenhuma o foi após o uso de tela ${ }^{19}$. Os mesmos achados foram encontrados por Khattab et al. (2020). Neste estudo, a recorrência foi identificada apenas no grupo de indivíduos que tiveram suas hérnias tratadas por sutura simples (2\%) e a IFO e o seroma foram mais frequentes entre os indivíduos tratados com o uso de tela ( $8 \%$ e $6 \%$ vs. $2 \%$ e $2 \%$, respectivamente) ${ }^{20}$.
Contudo, essas diferenças entre as técnicas de correção cirúrgica não foram estatisticamente significativas. Ademais, apesar da semelhança entre esses achados, o primeiro sugere a superioridade do uso de tela na correção cirúrgica das hérnias umbilicais e o segundo aponta a herniorrafia simples como a melhor estratégia de abordagem nos pacientes acometidos por esse defeito abdominal: Polat et al. atestam a utilidade das telas baseando-se no encontro de menores taxas de dor pós-operatória e, consequentemente, menores necessidades do uso de analgésicos; já Khattab et al. se apoiaram nos menores custos do procedimento sem o uso das próteses para fazer sua recomendação ${ }^{19,20}$.

\section{Tabela 3}

Características e principais resultados obtidos nos estudos incluídos.

\begin{tabular}{|c|c|c|c|c|c|c|c|}
\hline \multirow{2}{*}{ Autor } & \multirow{2}{*}{ Grupos } & \multirow{2}{*}{ Técnica } & \multirow{2}{*}{$\begin{array}{c}\mathbf{N}^{\circ} \mathrm{de} \\
\text { pacientes }\end{array}$} & \multicolumn{4}{|c|}{ Resultados } \\
\hline & & & & Recorrência & IFO & Hematoma & Seroma \\
\hline \multirow[t]{2}{*}{ Arroyo et al. } & Sutura & Sutura simples & 100 & $11(11 \%)$ & $3(3 \%)$ & $1(1 \%)$ & $5(5 \%)$ \\
\hline & Tela & $\begin{array}{l}\text { Tela de polipropileno } \\
\text { fixada no plano pré- } \\
\text { peritoneal }\end{array}$ & 100 & $1(1 \%)$ & $2(2 \%)$ & $1(1 \%)$ & $6(6 \%)$ \\
\hline \multirow[t]{2}{*}{$\begin{array}{l}\text { Kaufmann } \\
\text { et al. }\end{array}$} & Sutura & $\begin{array}{l}\text { Sutura simples } \\
\text { transversa }\end{array}$ & 138 & $17(12 \%)$ & $1(<1 \%)$ & $2(1 \%)$ & $1(<1 \%)$ \\
\hline & Tela & $\begin{array}{l}\text { Tela de polipropileno } \\
\text { fixada no plano pré- } \\
\text { peritoneal }\end{array}$ & 146 & $6(4 \%)$ & $3(2 \%)$ & $3(2 \%)$ & $5(3 \%)$ \\
\hline \multirow{2}{*}{$\begin{array}{l}\text { Khattab et } \\
\text { al. }\end{array}$} & Sutura & Sutura simples & 50 & $1(2 \%)$ & $1(2 \%)$ & - & $1(2 \%)$ \\
\hline & Tela & - & 50 & 0 & $4(8 \%)$ & - & $3(6 \%)$ \\
\hline \multirow[t]{2}{*}{ Polat et al. } & Sutura & Técnica de Mayo & 18 & $2(11 \%)$ & $1(5,5 \%)$ & $2(11 \%)$ & 0 \\
\hline & Tela & $\begin{array}{l}\text { Prolene Hernia System } \\
\text { (PHS) ou tela de } \\
\text { polipropileno fixada } \\
\text { na bainha anterior } \\
\text { do músculo reto } \\
\text { abdominal }\end{array}$ & 32 & 0 & $2(6 \%)$ & $2(6 \%)$ & $1(3 \%)$ \\
\hline
\end{tabular}




\section{DISCUSSÃO}

Em indivíduos hígidos, o manejo cirúrgico das hérnias umbilicais proporciona bons resultados. As principais complicações relacionadas a esse procedimento são seromas, hematomas e IFO, mais comuns quando as malhas cirúrgicas são utilizadas, e complicações respiratórias e cardiovasculares também podem ocorrer, sendo que, essas últimas, implicam internações prolongadas. Ademais, o tamanho da hérnia, a idade do indivíduo e a presença de comorbidades são os principais fatores relacionados à morbimortalidade da cirurgia.

São múltiplas as possibilidades de abordagem das hérnias umbilicais - aberta ou laparoscópica, com ou sem uso de tela, com ou sem fechamento do defeito herniário - e a escolha que orienta a sua correção deve considerar vários fatores, inclusive os detalhes que se referem à hérnia em si, principalmente os tamanhos do orifício e do saco herniários ${ }^{1}$. Nos últimos anos, a herniorrafia pela técnica de Mayo foi o método mais utilizado no reparo das hérnias umbilicais e, apesar das demonstradas altas taxas de recorrência associadas a essa metodologia, devido à ausência de alternativas comprovadamente eficazes, ela continua sendo a escolha de muitos cirurgiões. Todavia, o uso corriqueiro de malhas cirúrgicas no manejo de hérnias inguinais, femorais e incisionais, com baixas taxas de recorrência, trouxe à tona a possibilidade de aproveitamento dessa técnica também para a correção das hérnias umbilicais ${ }^{17}$. Do ponto de vista estrutural, a principal meta do reparo da hérnia consiste em evitar ou diminuir a possibilidade de que o conteúdo da cavidade abdominal volte a se insinuar através do defeito da parede. Assim, independentemente da técnica escolhida pelo cirurgião, em todos os casos, as suturas devem ser capazes de resistir à tensão exercida pela pressão intra-abdominal, garantindo a integridade da parede do abdome até que a cicatrização se estabeleça, evitando-se rupturas e recidivas ${ }^{21}$.

A tela sintética é o principal instrumento ao dispor dos cirurgiões capaz de reduzir as recidivas. Existe uma grande variedade de malhas disponíveis para uso nas correções dos defeitos herniários, porém a tela confeccionada a partir de polipropileno é a mais utilizada nesses procedimentos, desde a sua introdução no mercado. Entretanto, ao contrário do que se tem estabelecido para o manejo da hérnia inguinal, ainda não existe um consenso quanto à melhor escolha no reparo das hérnias umbilicais. Além disso, vale ressaltar que, apesar dos benefícios significativos associados ao uso dessas próteses exógenas, devem ser consideradas também as complicações a elas associadas.

O reparo através de sutura, a estratégia clássica, é um método heterogêneo, haja vista a variedade de técnicas passíveis de serem utilizadas na correção do defeito, e acarreta uma elevada taxa de recorrência. Contudo, apresenta menores custos e menor taxa de complicações pós-operatórias. Já a aplicação de tela, que também é um procedimento complexo - já que envolve, além das habilidades técnicas, a escolha do material e do design da prótese e o seu local de fixação - implica menor taxa de recorrência, porém, gera um maior potencial de complicações, essas relacionadas à prótese, como contratura da tela, infecção e dor crônica, que, todavia, têm baixa incidência. Vale ressaltar ainda que, apesar do maior custo do procedimento quando se utiliza a prótese, os custos associados a uma nova cirurgia, devido à recorrência, são também consideráveis ${ }^{1}$.

Com exceção de um estudo analisado, todos recomendam que o reparo com malha cirúrgica deve ser realizado, preferencialmente, nos pacientes com hérnia umbilical cujo orifício tem diâmetro igual ou maior a $1 \mathrm{~cm}^{17-20}$. No entanto, permanecem as controvérsias relacionadas à abordagem das hérnias umbilicais de tamanho menor que 1 $\mathrm{cm}$. As discussões relativas a esse ponto residem na crença em que, por serem muito pequenas, o risco de recorrência, mesmo com a abordagem clássica, seja semelhante ao risco com o uso de telas. Além disso, muitos se preocupam com a necessidade de se aumentar o defeito para que a prótese seja inserida ${ }^{18,22}$.

Em nossa prática, a escolha acerca da técnica a ser utilizada na correção da hérnia umbilical é individualizada e considera características como presença de comorbidades, IMC, reserva funcional e necessidade de alto desempenho em atividades cotidianas, além do tamanho do defeito herniário. Em geral, para pacientes adultos e saudáveis, damos sempre preferência ao uso de telas, assim como para os pacientes com hérnias cujo orifício apresenta diâmetro igual ou maior que $3 \mathrm{~cm}$ ou 
que apresentam hérnias recidivantes. Contudo, para aqueles que apresentam imunodeficiência, seja por uso de medicamentos ou resultante de alguma condição prévia, a indicação do uso de tela, principalmente no reparo de hérnias com orifícios menores que $3 \mathrm{~cm}$, é feita de forma mais criteriosa. Quando optamos pelo manejo cirúrgico com prótese nas hérnias de orifício pequeno, fazemos a fixação intraperitoneal da malha, o que tem permitido evitar o aumento do orifício herniário para a sua inserção e fixação.

A presente revisão teve algumas limitações. Primeiramente, apenas um estudo apresentava baixo risco de viés, sendo a qualidade metodológica da maioria dos estudos incluídos considerada intermediária. Em segundo lugar, não foram consideradas todas as variedades de telas disponíveis no mercado, nem foram levadas em conta as diferentes técnicas de fixação das próteses à parede abdominal, detalhes que podem ser relevantes para a execução dessa estratégia de correção da hérnia umbilical e para a obtenção dos bons resultados. Por fim, a pesquisa foi limitada aos artigos publicados na língua inglesa, o que pode ter introduzido um viés a este estudo. No entanto, vale ressaltar que, uma vez que a busca foi realizada em várias bases de dados, esse risco pode ter sido significativamente reduzido.

\section{CONCLUSÃO}

Evidências apontam a superioridade do uso de malhas cirúrgicas no reparo das pequenas hérnias umbilicais primárias, devido bons resultados observados quanto à redução da taxa de recorrência dessas hérnias após o uso dessa abordagem, podendo-se dizer que o uso das próteses pode se tornar o seu tratamento de escolha. Entretanto, o papel do uso das telas no manejo das hérnias umbilicais primárias com orifícios herniários de diâmetro igual ou superior a $1 \mathrm{~cm}$ e inferior a $3 \mathrm{~cm}$ permanece incerto, sendo necessários mais estudos.

\section{REFERÊNCIAS}

1. Earle DB, McLellan JA. Repair of umbilical and epigastric hernias. Surg Clin North Am 2013; 93:1057.
2. Gerdy PN: Nouvelles opérations pour guérir radicalement les hernies du ventre. Gaz Hôp 1:10, 1836.

3. Rutkow IM. The Perfix plug repair for groin hernias. Surg. Clin North Am. 2003; 83 (5): 1079-98.

4. Lichtenstein IL, Shore JM: Simplified repair of femoral and recurrent inguinal hernias by a "plug" technique. Am J Surg 128:439, 1974

5. Bendavid R. The merits of the Shouldice repair. Probl Gen Surg, 1995; 12: 105-9

6. Devlin HB, Kingsnorth A. Management of abdominal hernias. 2nd ed. London, Chapman \& Hall Medical, 1998

7. Berliner SD. Adult inguinal hernia; pathophysiology and repair. Surg Ann, 1983: 307-29.

8. Kingsnorth AN, Gray MR, Nott DM. Prospective randomized trial comparing the Shouldice technique and plication darn for inguinal hernia. $\mathrm{Br}$ J Surg, 1992; 79: 1068-70

9. Read RC. Influence of a relaxing incision on suture tension in Bassini's and Mcvay's repairs. Arch Surg, 1981; 116: 440-5

10. Usher FC, Ochsner J, Tuttle LD: Use of Marlex mesh in the repair of incisional hernias. Am Surg 24:969, 1958

11. Robbins AW, Rutkow IM. Mesh plug repair and groin hernia surgery. Surg Clin North Am 1998; 78: 1007-23

12. Bringman S, Ramel S, Heikkinen TJ. Tension-Free Inguinal Hernia Repair: TEP Versus Mesh-Plug Versus Lichtenstein- A Prospective Randomized Contolled Trial. Annals of Surgery, vol 237, No. 1, 142-147

13. Mottin CC, Ramos RJ, Ramos MJ. Utilização do sistema prolene de hernia (PHS) para o reparo de hérnias inguinais. Rev Bras Cir. [periódico na internet] 2011; 38(1).

14. Mayagoitia JC. Inguinal hernioplasty with the prolene hernia system. Hernia 2004;8(1):64-6

15. Millikan KW, Doolas A. A long-term evaluation of the modified mesh-plug hernioplasty in over 2,000 patients. Hernia (2008) 12:257-260

16. Higgins JP, Altman DG, Gotzsche PC et al (2011) The Cochrane collaboration's tool for assessing risk of bias in randomised trials.BMJ 343:d5928

17. Arroyo A, García P, Pérez F, et al. Randomized clinical trial comparing suture and mesh repair of umbilical hernia in adults. Br J Surg 2001; 88:1321.

18. Kaufmann R, Halm JA, Eker $\mathrm{HH}$, et al. Mesh versus suture repair of umbilical hernia in adults: a randomised, double-blind, controlled, multicentre trial. Lancet 2018; 391:860.

19. Polat C, Dervisoglu A, Senyurek G, Bilgin M, Erzurumlu $\mathrm{K}$, Ozkan K. Umbilical hernia repair with the prolene herniasystem. Am J Surg 2005; 190: 61-64. 
20. Khattab AM, Abdallah AS, Elbalshy MA, Albatanoney AA. Comparative study between herniorrhaphy alone versus hernioplasty in small-sized paraumbilical hernia. Int Surg J 2020;7:31-5.

21. Gianlupi A, Trindade MRM. Comparação entre o uso de fio inabsorvível (polipropileno) e fio absorvível (poliglactina 910) na fixação de prótese de polipropileno em correção de defeitos músculo-aponeurótico da parede abdominal. Estudo experimental em ratos. Acta Cir Bras. 2004;19(2): 94-102.

22. Holihan JL, Hannon C, Goodenough C, et al. Ventral Hernia Repair: A Meta-Analysis of Randomized Controlled Trials. Surg Infect (Larchmt) 2017; 18:647.

\section{Contribuição dos autores}

CAB: concepção e desenho da pesquisa, obtenção de dados e redação do manuscrito. DCO: obtenção de dados, redação do manuscrito e revisão crítica do manuscrito. MMA: análise e interpretação dos dados, redação do manuscrito, revisão crítica do manuscrito. MFG: análise e interpretação dos dados, redação do manuscrito, revisão crítica do manuscrito.

\section{Declaração de conflito de interesses}

Os autores declaram não haver conflitos de interesses.

\section{Fontes de financiamento}

Não houve.

\section{Agradecimentos}

Ao professor Alcino Lázaro da Silva, a nossa imensa gratidão pelo extenso e rico legado e por ser fonte de inspiração para o constante crescimento.

Autor correspondente:

Cirênio de Almeida Barbosa

cireniobarbosa@gmail.com

Editor:

Prof. Dr. Marcelo Riberto

Recebido em: 08/07/2020

Aprovado em: 20/10/2020 